\title{
ADVANCED MULTI-PHOTON CHROMOPHORES FOR BROAD-BAND ULTRA-FAST OPTICAL LIMITING
}

Aleksander Rebane

MONTANA STATE UNIV BOZEMAN

$11 / 04 / 2014$

Final Report

DISTRIBUTION A: Distribution approved for public release.

Air Force Research Laboratory

AF Office Of Scientific Research (AFOSR)/ RTD

Arlington, Virginia 22203

Air Force Materiel Command 


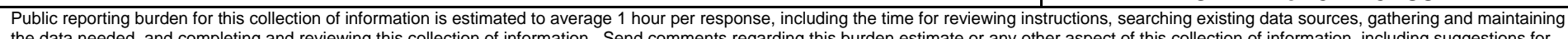

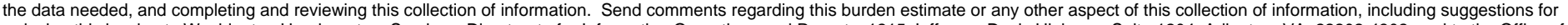

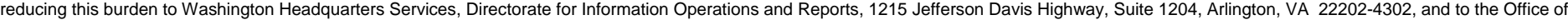
Management and Budget, Paperwork Reduction Project (0704-0188), Washington, DC 20503

\begin{tabular}{|l|l|}
\hline 1. AGENCY USE ONLY (Leave & 2. REPORT DATE \\
\hline
\end{tabular} blank) $10 / 31 / 14$

4. TITLE AND SUBTITLE ADVANCED MULTI-PHOTON CHROMOPHORES FOR BROAD-BAND ULTRAFAST OPTICAL LIMITING

\section{AUTHOR(S)}

Professor Aleksander Rebane

\section{PERFORMING ORGANIZATION NAME(S) AND ADDRESS(ES)}

Physics Department Montana State University EPS Building Bozeman, MT 59717

\section{SPONSORING I MONITORING AGENCY NAME(S) AND ADDRESS(ES)}

Charles Y-C Lee

CIV USAF AFMC AFOSR/RTD

Suite 325, Room 3112

Arlington, VA 22203-1954
Tel: (406) 9947831

Fax: (406) 9944452

Email: Rebane@physics.montana.edu
Email: charles.lee.21@us.af.mil

Fax: (703) 696-8449

Phone: (703) 696-7779

\section{SUPPLEMENTARY NOTES}

Purchase Request No.
3. REPORT TYPE AND DATES COVERED

Final Technical Report 3/1/09 - 7/31/14

5. FUNDING NUMBERS

Grant No. FA 9550-09-1-0219
PERFORMING ORGANIZATION REPORT NUMBER

\section{ABSTRACT (Maximum 200 Words)}

The goal is to design, synthesize and fully characterize new multi-functional organic chromophores that show high two photon absorption (2PA) cross section and high excited state absorption (ESA) efficiency, and thus provide for greatly improved optical power limiting functionality in a broad range of wavelengths. We optimize 2PA and ESA simultaneously by combining different chromophore modules by using $\pi$-conjugating linkers. The modular approach takes advantage of cooperative enhancement effect whereby the combined chromophore acquires much larger nonlinear absorption than the sum of the component chromophores. This allows us to greatly improve key photophysical properties, such as fast response time, high multi-photon absorption efficiency as well as chromophore solubility for high concentration materials. We also strive to improve our ability to project expected nonlinear-optical properties of new chromophores by testing validity limits of few essential state models with the ultimate goal of developing practical guidelines for designing new materials.

\begin{tabular}{|c|c|c|c|}
\hline \multicolumn{3}{|l|}{ 14. SUBJECT TERMS } & $\begin{array}{l}\text { 15. NUMBER OF PAGES } \\
\text { 16. PRICE CODE }\end{array}$ \\
\hline $\begin{array}{l}\text { 17. SECURITY CLASSIFICATION } \\
\text { OF REPORT }\end{array}$ & $\begin{array}{l}\text { 18. SECURITY CLASSIFICATION } \\
\text { OF THIS PAGE }\end{array}$ & $\begin{array}{l}\text { 19. SECURITY CLASSIFICATION } \\
\text { OF ABSTRACT }\end{array}$ & 20. LIMITATION OF ABSTRACT \\
\hline NSN 7540-01-280-5500 & & & $\begin{array}{l}\text { ndard Form } 298 \text { (Rev. 2-89) } \\
\text { cribed by ANSI Std. Z39-18 } \\
102\end{array}$ \\
\hline
\end{tabular}




\section{Final Technical Report 3/1/09 - 7/31/14}

Grant No. FA9550-09-1-0219

ADVANCED MULTI-PHOTON CHROMOPHORES FOR BROAD-BAND ULTRA-FAST OPTICAL LIMITING

A. Rebane, Physics Department, Montana State University, Bozeman, MT 59717

Tel. (406) 994 7831, Fax: (406) 994 4452, Email: rebane@physics.montana.edu

\section{Objectives (not changed)}

This project pursues both practical goals as well as broader fundamental and theoretical objectives. The practical goal is to design, synthesize and fully characterize new multi-functional organic chromophores that show simultaneously high 2PA cross section and high ESA efficiency in a broad range of overlapping wavelengths, and thus provide basis for new materials with greatly improved OPL functionality. One specific goal is to optimize 2PA and ESA simultaneously, in the same overlapping wavelength range. For this, we combine different chromophore modules by $\pi$-conjugating links. The modular approach takes advantage of our ability to predict $2 \mathrm{PA}$ and ESA properties of the conjugated constructs based on the known properties of the units. This allows greatly improving the key photo physical properties, such as fast response time, high multi-photon absorption efficiency as well as chromophore solubility for high concentration materials. Our approach will also streamline the synthetic effort by providing more rational design principles. Broader objective is to improve quantitative understanding of the 2PA and ESA processes, and through this achieve more accurate description of NLO material performance. We compare the measured absolute 2PA cross sections and spectra of various organic molecules with those obtained from the few (two or three) essential states model, assuming certain parameter values that are either previously calculated theoretically or measured. We study conditions under which the two- or three-level models are applicable for quantitative description of 2PA, and estimate the corresponding maximum realistic attainable peak 2PA cross sections and spectral widths. Based on our observations we formulate the steps required to increase the intensity and broaden the spectral coverage of instantaneous 2PA by optimizing intrinsic molecular parameters such as transition dipole moments, permanent dipole moments and excited states energies.

\section{Accomplishments \& New Findings}

\subsection{Summary of main findings (Abstract)}

1. We have explored following series of platinum acetylides with different substitution- and construction motifs showing high ultrafast optical nonlinearity studied the relevant 2PA spectra vs structure relations (joint effort with U of Florida team Kirk Schanze, Galyna Dubinina and AFRL Tom Cooper group):

i.) Record high 2PA in phenylene vinylene (PPV) platinum(II) acetylides. We designed, synthesized and carried out a detailed photon physical study of a new series of Pt acetylides complexes based on 4,4'-bis(diphenylamino) stilbene (BDPAS) and 4,4'-bis(diphenylamino) distyrylbenzene (BDPADSB) structural. The complexes exhibit in THF solution very high twophoton absorption (peak cross section values $\sigma_{2} \sim 10^{4} \mathrm{GM}$ ) as measured both by femtosecond 2- 
photon excited fluorescence (2PEF) method as well as by the newly developed femtosecond nonlinear transmission (NLT) method. The prodigious 2PA cross sections span a broad range of wavelengths, $570-810 \mathrm{~nm}$, which overlaps favorably with the triplet excited state absorption, thus facilitating efficient dual-mode power limiting [1-3]. The properties were preserved in PMMA monoliths at moderate concentratioins [4].

ii.) Inversion symmetry breaking in nominally quasi-centrosymmetric fluoren-based Ptcomplexes. trans-bis-(tributylphosphine)-bis- (4-((9,9-diethyl-7-ethynyl-9H-fluoren-2-yl) ethynyl)-R)-platinum complexes, where eleven different substituents, $\mathrm{R}=\mathrm{N}(\text { phenyl })_{2} \quad\left(\mathrm{NPh}_{2}\right)$, $\mathrm{NH}_{2}, \mathrm{OCH}_{3}$, t-butyl, $\mathrm{CH}_{3}, \mathrm{H}, \mathrm{F}, \mathrm{CF}_{3}, \mathrm{CN}$, benzothiazole (BT) and $\mathrm{NO}_{2}$, represent a range of electron-donating (ED) and electron-withdrawing (EW) strengths, while the Pt-core acts as a weak ED group. 2PA cross section in the 540-810 nm excitation wavelength range measured by complementary femtosecond $2 \mathrm{PEF}$ and NLT methods show that the peak $2 \mathrm{PA}$ cross sections of neutral- and ED-substituted Pt-complexes occur at $\mathrm{S}_{0} \rightarrow \mathrm{S}_{\mathrm{n}}$ transitions to higher energy states, above the lowest-energy $\mathrm{S}_{0} \rightarrow \mathrm{S}_{1}$ transition, and the corresponding values increase systematically with increasing ED strength, reaching maximum value, $\sigma_{2} \sim 300 \mathrm{GM}\left(1 \mathrm{GM}=10^{-50} \mathrm{~cm}^{4} \mathrm{~s}\right)$ for $\mathrm{R}$ $=\mathrm{NPh}_{2}$. At transition energies overlapping with the lowest-energy $\mathrm{S}_{0} \rightarrow \mathrm{S}_{1}$ transition in the onephoton absorption (1PA) spectrum, the same neutral- and ED-substituted Pt-complexes show weak 2PA, $\sigma_{2}<30-100 \mathrm{GM}$, which is in agreement with the nearly quadrupolar structure of these systems. Surprisingly, EW-substituted Pt-complexes display a very different behavior, where the peak 2PA of the $\mathrm{S}_{0} \rightarrow \mathrm{S}_{1}$ transition gradually increases with increasing EW-strength, reaching value up to, $\sigma_{2}=700 \mathrm{GM}$ for $\mathrm{R}=\mathrm{NO}_{2}$, while in the $\mathrm{S}_{0} \rightarrow \mathrm{S}_{\mathrm{n}}$ transition region the peak $2 \mathrm{PEF}$ cross section decreases, indicating breaking of inversion symmetry $[5,6]$.

iii.) 2,5-bis(dodecanoxy) phenyleneethynylene-butadiyne (DAc) pt-complexes with 2, 4, 6 and 8 phenyl rings (synthesis Tom Cooper/AFRL and other international collaborators); The largest cross-section (5000 GM) was obtained for the DAc octamer [7];

iv.) Au(I) and Pt(II) acetylide complexes of a thienyl-carbazole chromophore (Kirk Schanze)

$\pi$-conjugated oligomers of the type ET-CBZ-TE $(\mathrm{E}=$ ethynylene, $\mathrm{T}=2,5$-thienylene, $\mathrm{Cbz}=3,6$ carbazole) that feature $\mathrm{Au}(\mathrm{I})$ or $\mathrm{Pt}(\mathrm{II})$ acetylide "end groups", as well as a $\mathrm{Pt}(\mathrm{II})$-acetylide linked polymer (CBZ-Au-1 and CBZ-Pt-1, CBZ-Poly-Pt). Peak 2PA cross section measured by NLT method reached 800-1000 GM at $550 \mathrm{~nm}$ [8];

v.) We studied the 2PA spectra of a series of linear Pt-capped phenylene ethynylene oligomers (synthesis Kirk Schanze)[9];

vi.) Comparison of cis and trans stereoisomers of Pt-core phenylethynyl phenylethynylene (PE2), diphenylaminofluorene (DPAF), and benzothiazolefluorene (BTF) (synthesis Kirk Schanze) [10]. 2PA spectra measured by NLT method show that both isomers show similar 2PA spectra and peak cross section values;

2. New near collimated beam technique for high-accuracy measurement of femtosecond nonlinear transmission. We have developed a newexperiment for measuring the intensitydependent transmission of 2PA chomophores in a broad range of wavelengths. This technique offers a very high accuracy ( $\sim 0.02 \%$ transmission change) and is of particular importance for 
characterization of the 2PA in Pt-complexes and other compounds with low fluorescence emission. As a preliminary demonstration of this new technique we have measured the intrinsic 2PA spectra and the 2PA cross sections of some known chromophores that have been previously characterized by the fluorescence method.

3. We have explored structure-property relationships in an extensive library of porphyrins and porphyrin dimers with expanded $\pi$-system. This data provides crucial insight into the push-pull 2PA-enhancement mechanism. Following systems were studied in detail:

i.) Amplified two-photon absorption in trans-A2B2-porphyrins bearing nitrophenylethynyl substituents [11]. We show that peripheral nitro groups enhance the maximum two-photon absorption cross section of trans-A2B2-porphyrins bearing two phenylethynyl substituents by more than one order of magnitude. Maximum values as high as $1000 \mathrm{GM}$ result from realization of suitable conditions for effective resonance enhancement along with a lowering of the energy and intensification of the two-photon allowed transitions in the Soret region;

ii.) Symmetrically- and non-symmetrically meso-substituted porphyrins with ultimate solubility $[12,13]$.

iii.) Fused porphyrin dimers (porphyrin tapes) synthesized by (Anderson, U. of Oxford) [14];

iv.) $\pi$-conjugated porphyrin oligomers and polymers [15] (Gruko, Anderson);

4. New model to explain vibronic enhancement in the 2PA spectra [16]. The model is based on Herzberg-Teller expansion of the permanent electric dipole moment difference, and allows to quantitatively explain why the peak 2 PA cross section of the first vibronic component $(0-1)$ is often higher in value than the corresponding vibration-less component (0-0) of the same electronic 2PA band. 


\subsection{Summary and detailed description of results}

3.2.1. Phenylene vinylene platinum(II) acetylides with prodigious 2-photon absorption [1,2].

A series of cross-conjugated platinum(II) acetylide complexes that contain extended $\mathrm{p}$-(phenylene vinylene) chromophores were synthesized and characterized. The complexes exhibit very high femtosecond 2-photon absorption (2PA) cross-section values ( $\sigma_{2 \mathrm{PA}}$ up to $\left.10,000 \mathrm{GM}\right)$, as measured by nonlinear transmission (NLT) and two-photon excited fluorescence (2PEF) methods. The large 2PA cross section values span a broad range of wavelengths, $570-810 \mathrm{~nm}$, and they overlap with the triplet excited state absorption. Spectral coincidence of high cross-section 2PA and triplet absorption is a key feature giving rise to efficient dual-mode optical power limiting (OPL). Fig. 1 shows 2PA spectra of seven new Pt-acetylide complexes (TPV0, TPV1, TPV2, TPV1-Ph, TPV2-T2, crossTPV1 and crossTPV3) corresponding three different ligand chromophores (TPV0-Ligand, TPV1-Ligand and TPV2-Ligand) measured by 2PEF method (red symbols) and NLT method (blue symbols). Linear extinction spectra(solid line) are shown for comparison. Note very good qualitative correspondence between the two measurement methods. TPV2, TPV1-Ph, TPV2-T2, crossTPV1 and crossTPV3 exhibit prodigious two-photon absorption (peak cross section values $\sigma_{2} \sim 10000 \mathrm{GM}$ ) in a broad range of wavelengths, $570-810 \mathrm{~nm}$, which also overlaps with the measured triplet-triplet excited state absorption, thus facilitating efficient dual-mode power limiting. To our knowledge these are the most efficient 2PA absorbing Ptcomplexes reported so far.
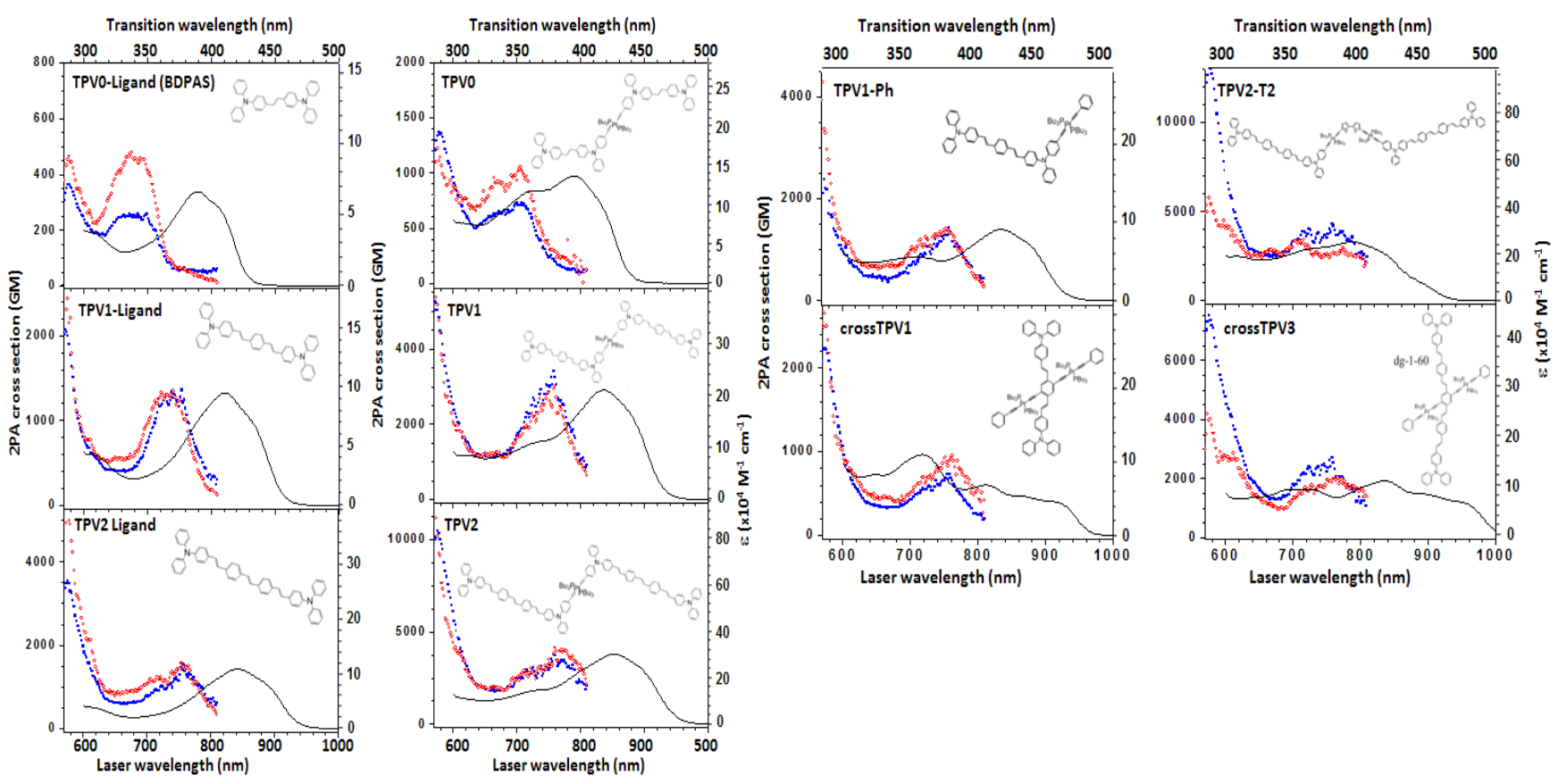

Fig. 1. 2PA spectra of seven new Pt-acetylide complexes (TPV0, TPV1, TPV2, TPV1-Ph, TPV2-T2, crossTPV1 and crossTPV3) corresponding ligand chromophores (TPV0-Ligand, TPV1-Ligand and TPV2Ligand) measured by 2PEF method (red symbols) and NLT method (blue symbols). Linear extinction spectra (solid line) are shown for comparison;

3.2.2. We studied instantaneous $2 \mathrm{PA}$ in a series of nominally quasi-centrosymmetric trans-bis(tributylphosphine)-bis-

(4-((9,9-diethyl-7-ethynyl-9H-fluoren-2-yl) ethynyl)-R)-platinum 
complexes, where eleven different substituents, $\mathrm{R}=\mathrm{N}$ (phenyl) $)_{2}\left(\mathrm{NPh}_{2}\right), \mathrm{NH}_{2}, \mathrm{OCH}_{3}, t$-butyl, $\mathrm{CH}_{3}, \mathrm{H}, \mathrm{F}, \mathrm{CF}_{3}, \mathrm{CN}$, benzothiazole (BT) and $\mathrm{NO}_{2}$, represent a range of electron-donating (ED) and electron-withdrawing (EW) strengths, while the Pt-core acts as a weak ED group. Fig. 2 shows the 2PA spectra measured in the 540-810 $\mathrm{nm}$ excitation wavelength range by complementary femtosecond two-photon excited fluorescence (2PEF) and nonlinear transmission (NLT) methods.
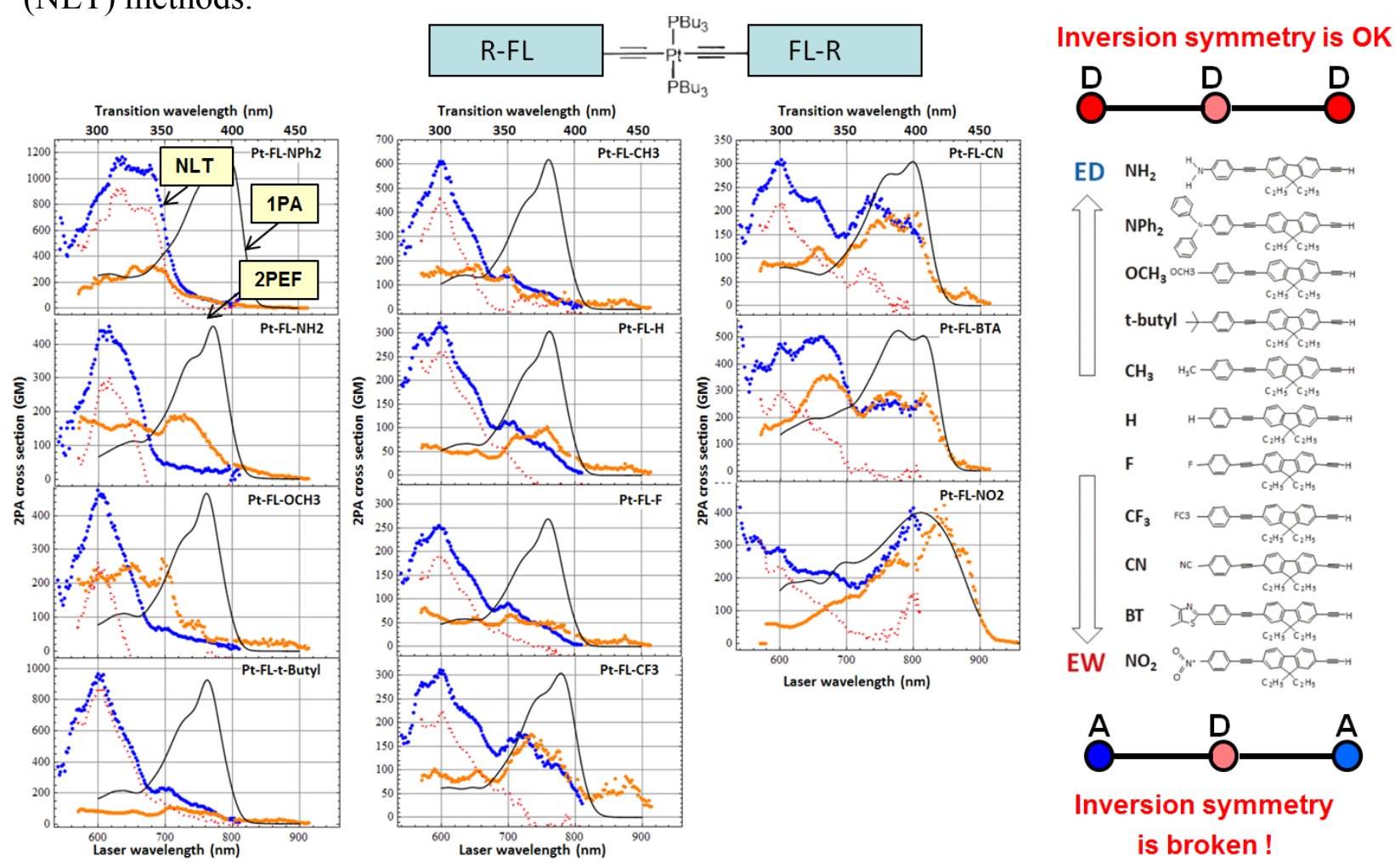

Fig. 2. 2PA spectra of eleven Pt-acetylide complexes measured by $2 \mathrm{PEF}$ method (orange symbols) and NLT method (blue symbols). Linear extinction spectra (solid line) are shown for comparison;

The peak 2PA cross sections of neutral- and ED-substituted Pt-complexes occur at $\mathrm{S}_{0} \rightarrow \mathrm{S}_{\mathrm{n}}$ transitions to higher energy states, above the lowest-energy $S_{0} \rightarrow S_{1}$ transition, and the corresponding values increase systematically with increasing ED strength, reaching maximum value, $\sigma_{2} \sim 300 \mathrm{GM}$ for $\mathrm{R}=\mathrm{NPh}_{2}$. At transition energies overlapping with the lowest-energy $\mathrm{S}_{0} \rightarrow \mathrm{S}_{1}$ transition in the one-photon absorption (1PA) spectrum, the same neutral- and EDsubstituted Pt-complexes show weak 2PA, $\sigma_{2}<30-100 \mathrm{GM}$, which is in agreement with the nearly quadrupolar structure of these systems. Surprisingly, EW-substituted Pt-complexes display a very different behavior, where the peak $2 \mathrm{PA}$ of the $\mathrm{S}_{0} \rightarrow \mathrm{S}_{1}$ transition gradually increases with increasing EW-strength, reaching value up to, $\sigma_{2}=700 \mathrm{GM}$ for $\mathrm{R}=\mathrm{NO}_{2}$, while in the $\mathrm{S}_{0} \rightarrow \mathrm{S}_{\mathrm{n}}$ transition region the peak $2 \mathrm{PEF}$ cross section decreases. We explained this effect by breaking of inversion symmetry due to conformational distortions associated with low energy barrier for ground state rotation of the ligands.

Our findings are corroborated by theoretical calculations that show large increase of the permanent electric dipole moment change in the $S_{0} \rightarrow S_{n}$ transition is possible when the ligands with strong EW substituents are twisted by $90^{\circ}$ relative to the planar chromophore. Fig. 3 correlates experimental and calculated (vacuum) permanent electric dipole moment change $\Delta \mu$ 
values. Good correlation indicates that a twist around just one bond may be responsible for already introducing enough distortion to break quasi-inversion symmetry. Other conformations not considered here may be also contributing to dipolar behavior of Pt-complexes with EWligands.

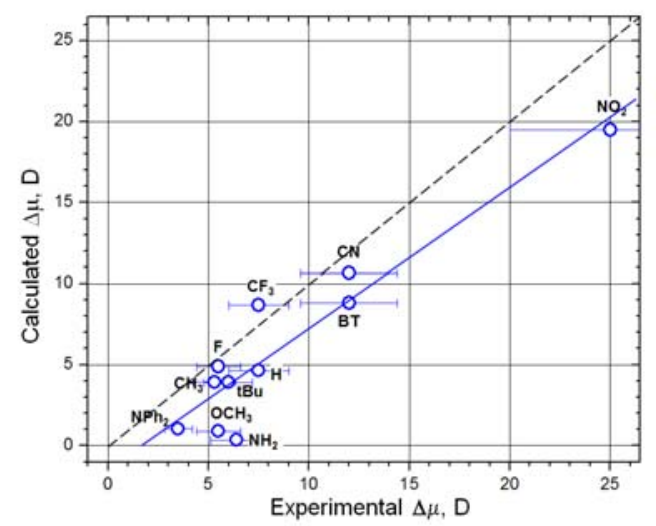

Fig. 3. Experimental (horizontal axis) and calculated vacuum (vertical axis) $\Delta \mu$ values for the lowest electronic transition of Pt-complexes. Linear fit is shown with solid line. Dashed line corresponds to exact correlation.

3.2.3. Fig. 4 shows the structure (left panel) and 2PA spectra (right panel) of 2,5-bis(dodecanoxy) phenyleneethynylene-butadiyne (DAc) series with 2,4,6 and 8 phenyl rings. The largest cross section (5000 GM at $740 \mathrm{~nm}$ ) is obtained for the DAc octamer [7].
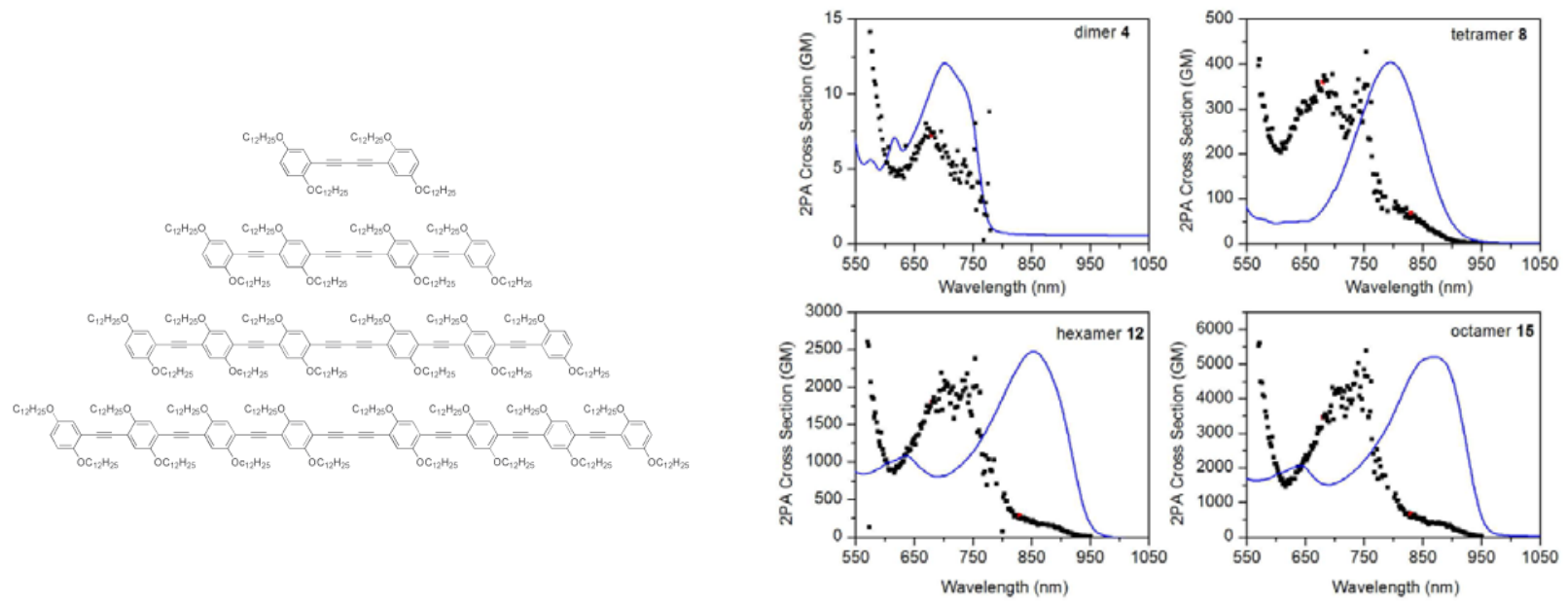

Fig. 4. Chemical structure (left panel) and 2PA spectra (symbols) of DAc series. Linear absorption spectrum (solid line) is shown for comparison. The 2PA is measured by $2 \mathrm{PEF}$ method.

3.2.4. $\mathrm{Au}(\mathrm{I})$ and $\mathrm{Pt}(\mathrm{II})$ acetylide complexes of a thienyl-carbazole (CBZ) chromophore [7] (synthesis Kirk Schanze group). To better understand the photophysics and NLO properties of carbazole containing $\pi$-conjugated oligomers of the type ET-CBZ-TE ( $E=$ ethynylene, $\mathrm{T}=2,5$ thienylene, $\mathrm{Cbz}=3,6$-carbazole), a detailed investigation was carried out on a series of oligomers that feature $\mathrm{Au}(\mathrm{I})$ or $\mathrm{Pt}(\mathrm{II})$ acetylide "end groups", as well as a $\mathrm{Pt}(\mathrm{II})$-acetylide linked polymer (CBZ-Au-1 and CBZ-Pt-1, CBZ-Poly-Pt). Fig. 5 shows the femtosecond 2PA spectra measured 
by the NLT method. The 2PA cross section in the degenerate $\mathrm{S}_{0} \rightarrow \mathrm{S}_{1}$ transition region is in the range 10-100 GM, and increased monotonically toward shorter wavelengths, reaching 800-1000 $\mathrm{GM}$ at $550 \mathrm{~nm}$;
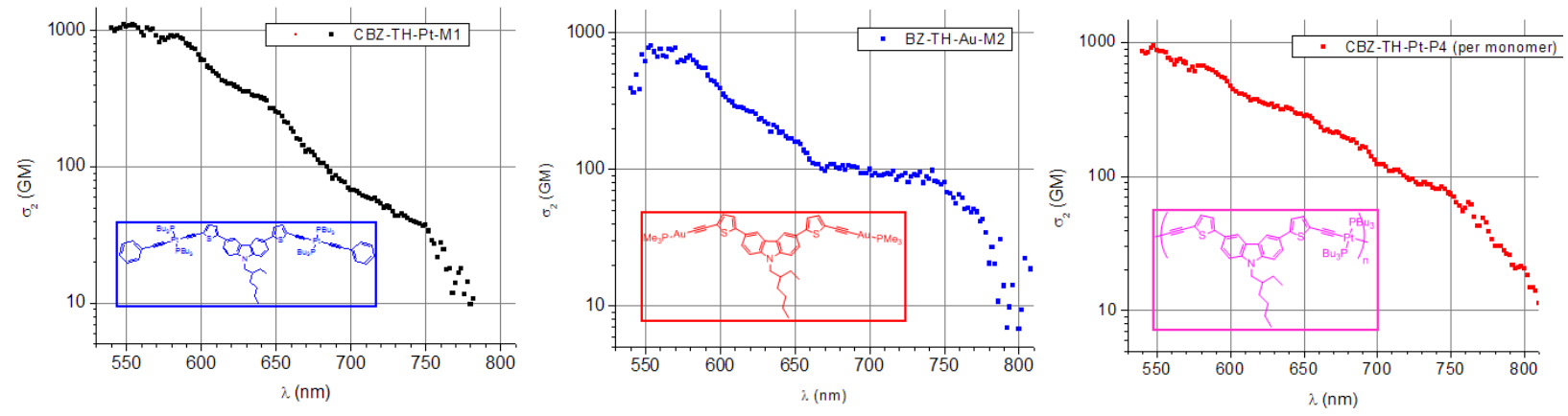

Fig. 5. Chemical structure (inserts) and 2PA spectra (symbols) of CBZ series in THF. The 2PA is measured by NLT method. Note that cross section values are given on logarithmic scale.

3.2.5. Platinum capped phenylene ethynylene oligomers (synthesis Kirk Schanze) [9]. Fig. 6 shows the 2PA spectra of linear $\mathrm{Ph}_{\mathrm{x}}-\mathrm{Pt}_{2}$ complexes measured with NLT method. First we observe that the maximum $\sigma_{2}$ increases more or less in proportion with the chain length and that the characteristic ratio, $\sigma_{2} /$ molecular mass $\sim 1$ (typical value for conjugated systems). Comparison of the 2PA and 1PA spectra and the fact that 2PA in the lowest $\mathrm{S}_{0}-\mathrm{S}_{1}$ transition is weak seems to indicate that the center of inversion is preserved. It is also interesting that the smallest chromophore in the series, $\mathrm{Ph}_{1} \mathrm{Pt}_{2}$, has higher 2PA activity than benchmark PE2 chromophore (one $\mathrm{Pt}$ in the middle with two $\mathrm{Ph}$ rings attached) that we have measured before with the same method.
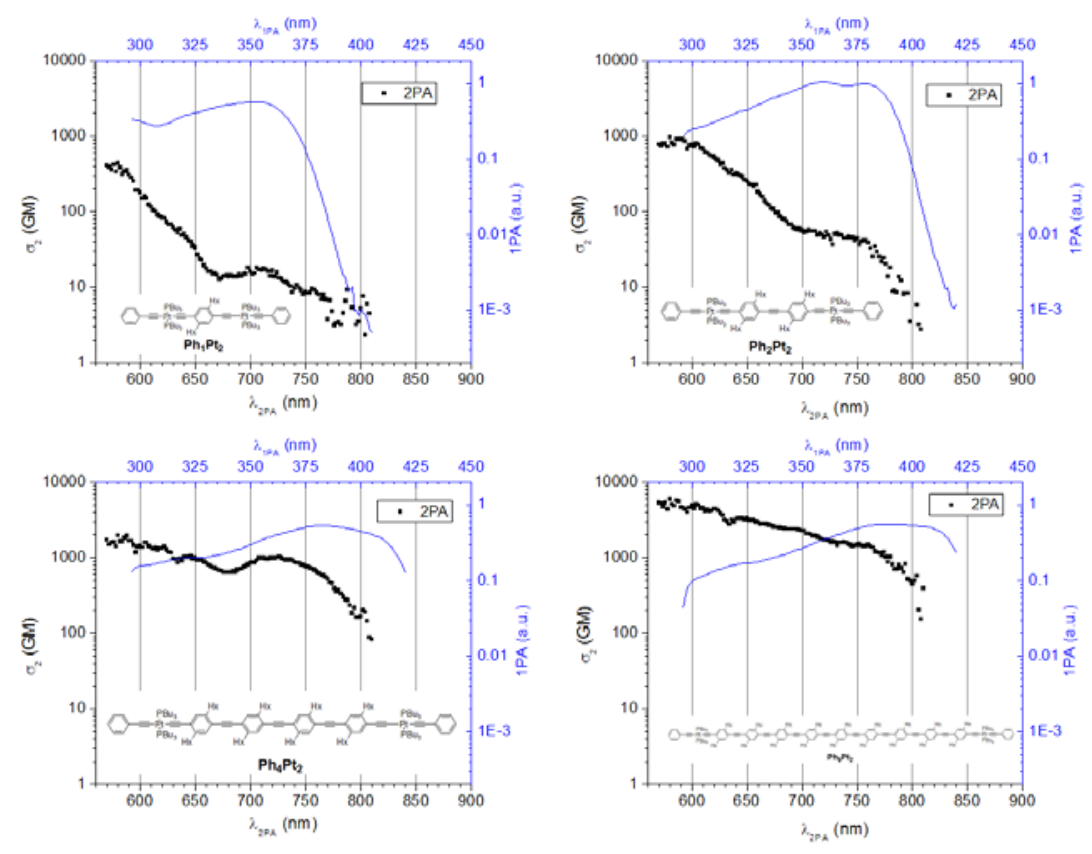

Fig. 6. Chemical structure (inserts) and $2 \mathrm{PA}$ spectra (symbols) of $\mathrm{Ph}_{\mathrm{x}}-\mathrm{Pt}_{2}$ oligomers measured by NLT method. Linear extinction spectra are shown for comparison. Note that 2PA cross sections are given in logarithmic scale. 
3.2.6. Pt-core stereoisomers. Three cis platinum(II) acetylide complexes: phenylethynylene (PE2), diphenylaminofluorene (DPAF), and benzothiazolefluorene (BTF) were characterized under one- and 2-photon conditions against the known trans analogs. The heavy metal platinum centers in both geometries give rise to efficient intersystem crossing to the triplet manifold. The peak cross section of the pronounced 2-photon allowed transition (Fig. 7) at $600-650 \mathrm{~nm}$ of the cis- DPAF and cis-BTF complexes is $\sigma_{2}=520 \mathrm{GM}$ and $\sigma_{2}=1200 \mathrm{GM}$. These values constitute respectively $40 \%$ and $85 \%$ decrease compared to the peak values in the corresponding transgeometries, which allows us to conclude that the stereochemistry does affect the conjugation through the Pt core.
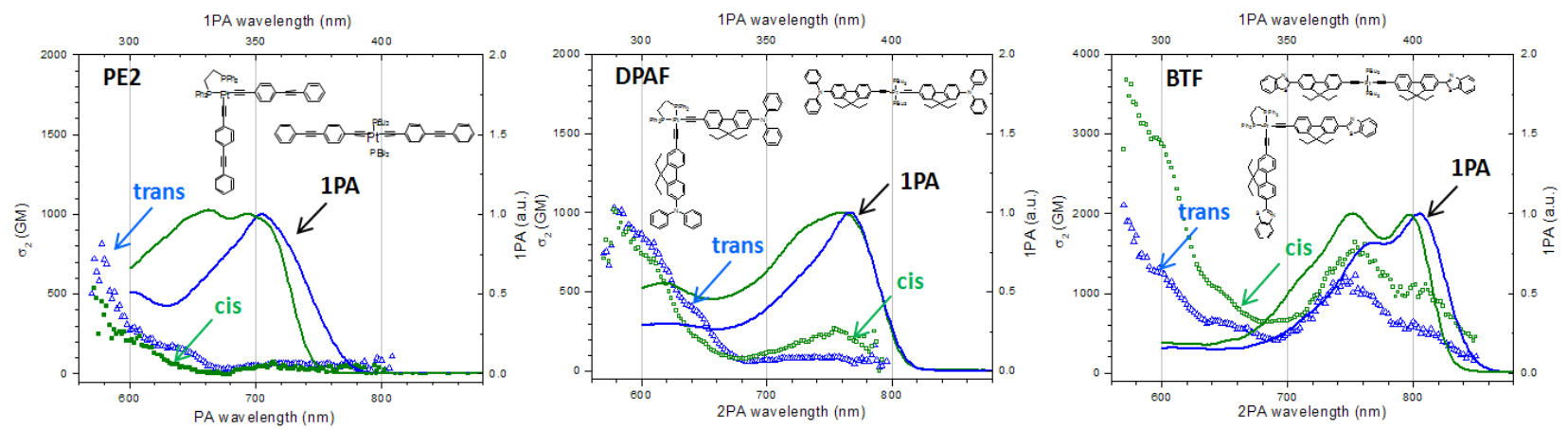

Fig. 7. Two-photon absorption spectra of cis- and trans-PE2, cis- and trans-DPAF and of cis- and transBTF (c-d) in THF at ambient conditions; Empty blue triangles correspond to "trans" isomer; Solid green squares - to "cis" isomer. Linear absorption spectra are shown for comparison by solid lines.

\subsubsection{Pt-PE1-R complexes series (synthesis Tom Cooper/AFRL).}
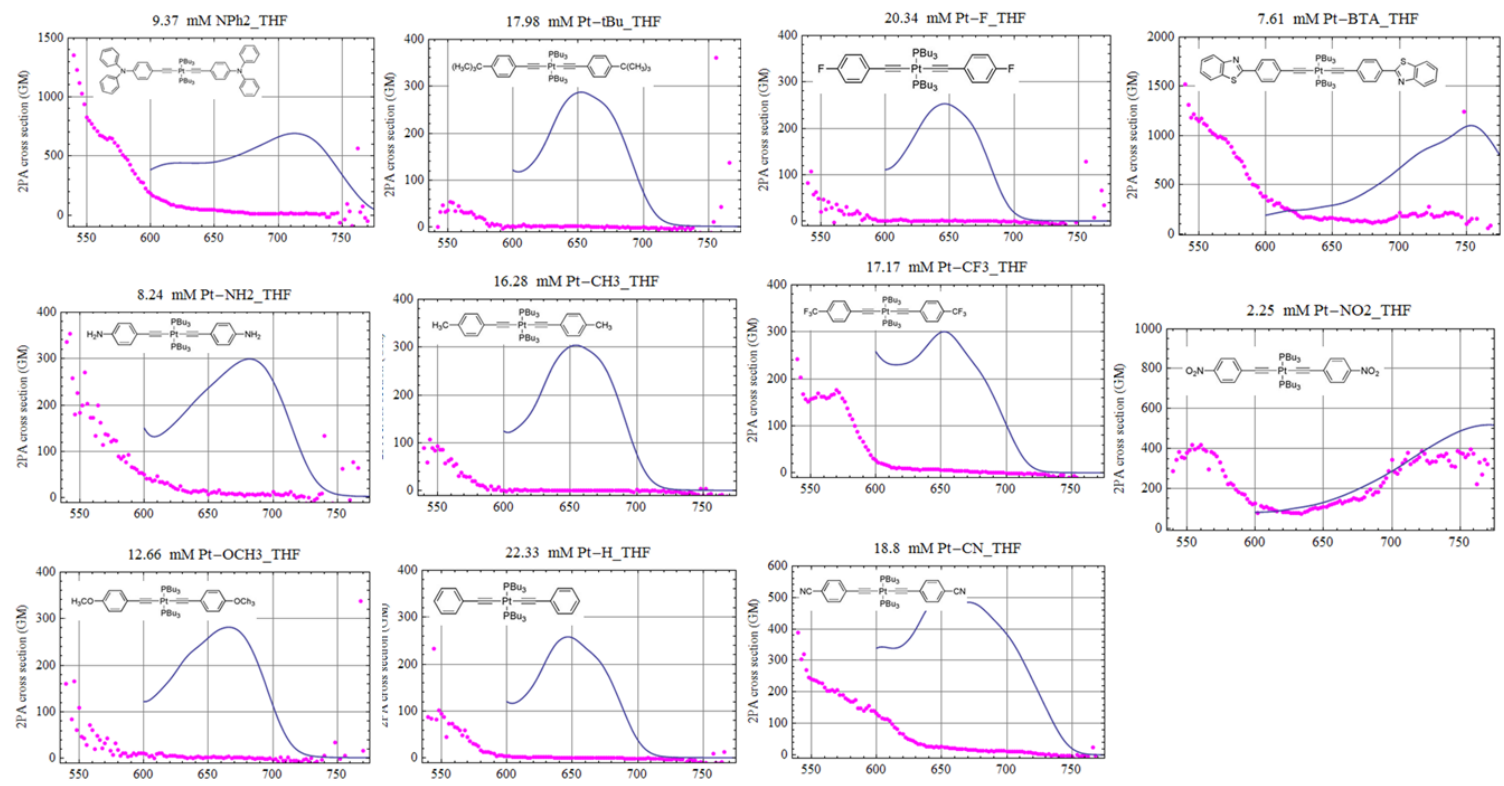

Fig. 8. Chemical structure (inserts) and 2PA spectra (symbols) of Pt-R series with 11 different substituents representing a systematic variation in electron donating (ED) and electron withdrawing (EW) strengths $\mathrm{R}$ $=\mathrm{NPh}_{2}, \mathrm{NH}_{2}, \mathrm{OCH}_{3}, \mathrm{t}-\mathrm{Butyl}, \mathrm{CH}_{3}, \mathrm{H}, \mathrm{F}, \mathrm{CF}_{3}, \mathrm{CN}$, Benzothiazole (BT) and $\mathrm{NO}_{2}$. Linear absorption (solid line) is shown for comparison. 
3.2.Femtosecond nonlinear transmission of thick solid samples $[1,4]$. Qualitative improvement of measuring intensity-dependent nonlinear transmission (NLT) was achieved by developing a new technique using nearly collimated (i.e. very long Rayleigh range) excitation laser beam. This allows measurements of samples with practically unlimited thickness. The larger beam size $(0.3$ $1 \mathrm{~mm}$ diameter) allows measuring samples that are not of ideal optical quality. We are currently able to measure NLT spectra in 540 - $1300 \mathrm{~nm}$ range. Fig. 9 shows the experimental scheme (left panel) and 2PA spectra (right panel) measured in a stack of solid polymer samples with total path length $\sim 3 \mathrm{~cm}$.
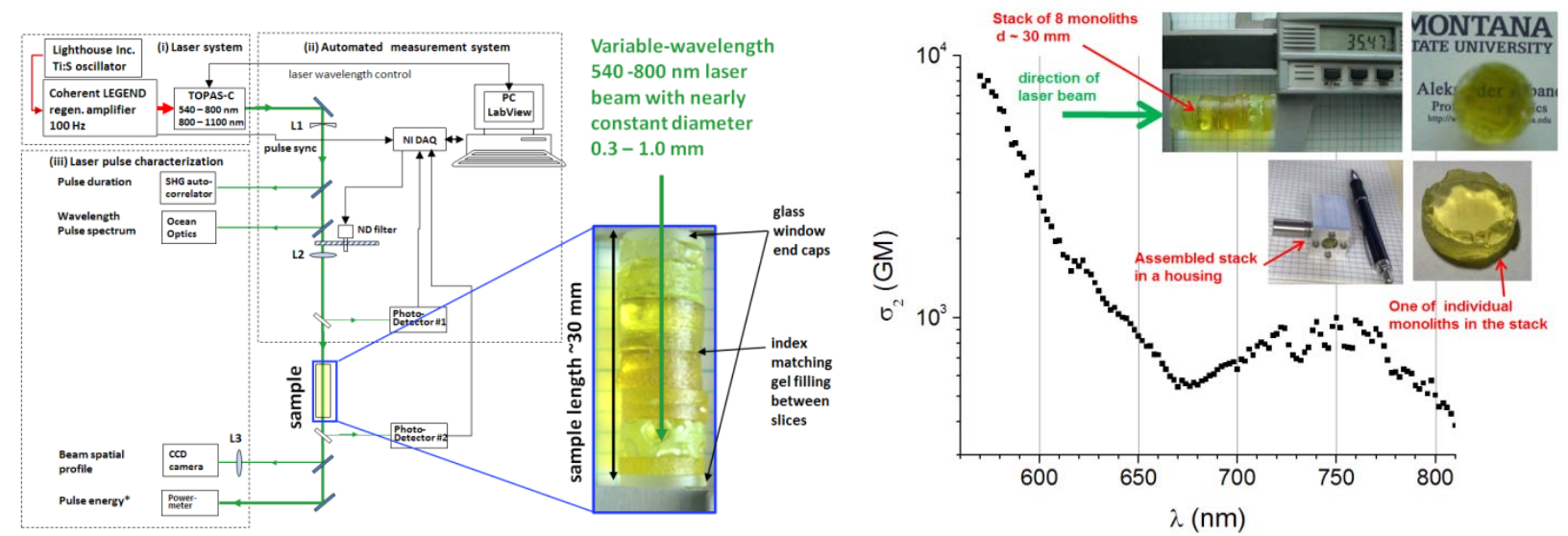

Fig. 9. Femtosecond 2PA measurement in thick solid sample. Left panel: Experimental setup comprising femtosecond optical parametric amplifier pumped by femtosecond Ti:S regenerative amplifier. Insert shows a stack of dye-doped polymer blocks with total thickness $\sim 3 \mathrm{~cm}$; Right panel: Effective two-photon absorption spectrum measured in the stack by scanning the fs laser wavelength in the range $540-810 \mathrm{~nm}$. Inset shows additional views of the polymer block samples.
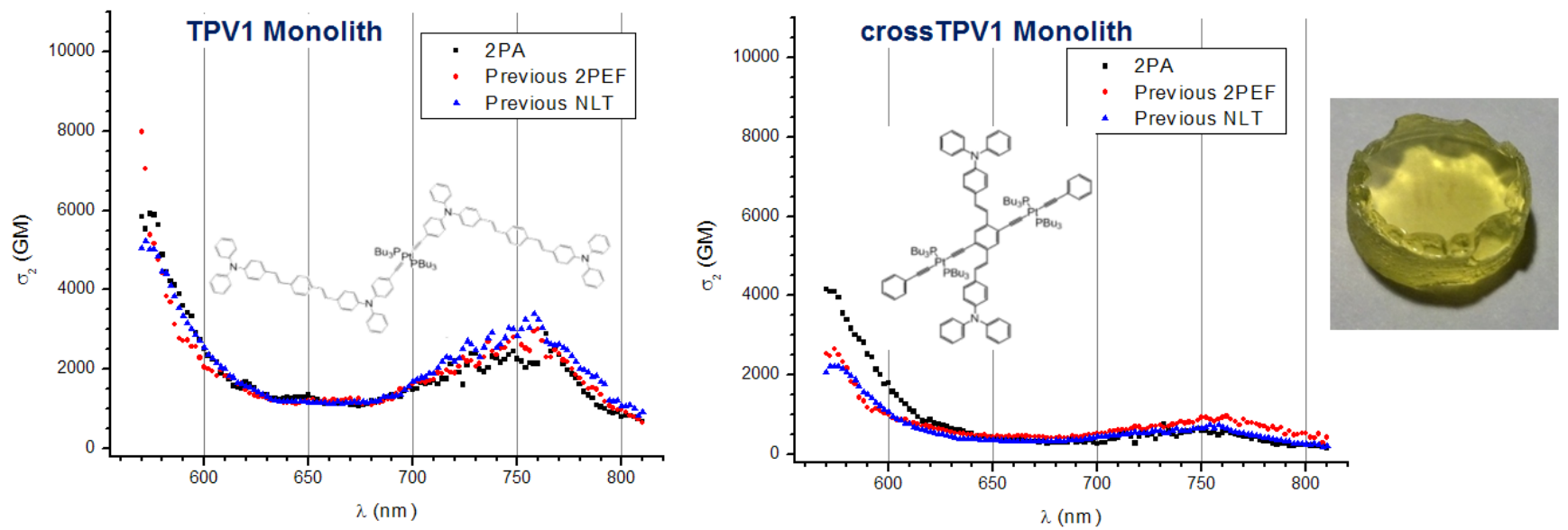

Fig. 10. Comparison of fs NLT measurement by NLT in activated PMMA monoliths (black symbols) with measurement in solution by NLT (blue symbols) and 2PEF (red symbols).

To conclude this subsection we note that, as a result of recent technical improvements in femtosecond 2PA spectroscopy, we are able to ease the bottleneck that has existed for years in characterizing newly synthesized compounds world-wide. 


\subsection{Conjugated porphyrins}

3.3.1. We show that peripheral nitro groups enhance the maximum two-photon absorption cross section of trans- $\mathrm{A}_{2} \mathrm{~B}_{2}$-porphyrins bearing two phenylethynyl substituents by more than one order of magnitude [11]. Maximum values as high as $1000 \mathrm{GM}$ result from realization of suitable conditions for effective resonance enhancement along with a lowering of the energy and intensification of the two-photon allowed transitions in the Soret region. The chromophores were synthesized by Daniel Gryko (Organic Chemistry Institute Polish Academy of Sciences) and Harry Anderson (University of Oxford). We are interested in the origin of the 2PA-allowed transitions that in many cases appear in the transition energy region above the Q-bands but below the Soret band. So far identification of these transitions has been a problem because as a rule in the corresponding 1PA spectra there are usually only very weak features. Fig. 11 shows the 2PA spectra and the cross sections of four new porphyrins with phenyl moiety attached through triple bond in two opposite meso-positions. The 2PA data shows no strong features in the $900-1100 \mathrm{~nm}$ laser wavelength region. This shows that attaching two phenylene-acetylene groups alone does not lead to the characteristic changes in the 2PA that we do observe when thriphenylamino ending groups are attached. Note however that symmetrical end-capping with $\mathrm{EW}$ groups $\left(\mathrm{NO}_{2}\right)$ enhanced the $2 \mathrm{PA}$ by an order of magnitude at $850-900 \mathrm{~nm}$.
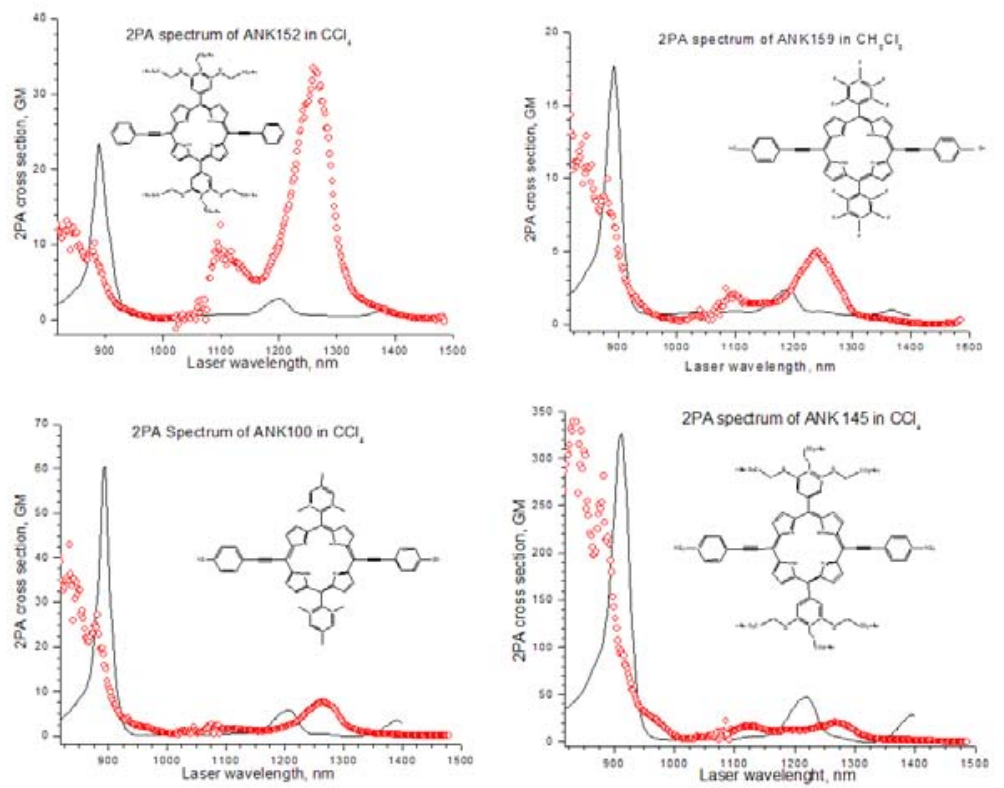

Fig. 11. 2PA spectra (symbols) and 1PA spectra (solid line) in four symmetrically meso-substituted porhyrins.

3.3.2. Our goal was to explore porphyrins with extreme solubility $[12,13]$. For this purpose a large and diverse library of trans-A2B2 and A2BC-porphyrins possessing two arylethynyl substituents at the meso positions was synthesized. All compounds fall in three general types A- $\pi-A, D-\pi-D$ or $\mathrm{D}-\pi-\mathrm{A}$, where $\mathrm{A}$ is an electron-acceptor and $\mathrm{D}$ is an electron-donor moiety. These porphyrins contain two polyalkoxyaryl substituents, resulting in very low melting points (typically 110$125^{\circ} \mathrm{C}$ ) and superb solubility in non-polar solvents. Some of these porphyrins exhibit two different crystal phases in addition to an isotropic liquid state. Their linear and nonlinear optical properties were thoroughly elucidated and analyzed. $\pi$-Extended porphyrins emit light in the NIR 
and have moderate triplet state lifetimes. The increase of 2PA cross-section in the Soret region for porphyrins bearing strongly electron-donating groups has been attributed to resonance enhancement (caused by intensification, red-shift and broadening of lowest Q-band) of geradegerade transition. The combination of high two-photon absorption cross-section and low melting points makes them perfect candidates for nonlinear optical materials for the 600-900 $\mathrm{nm}$ range.
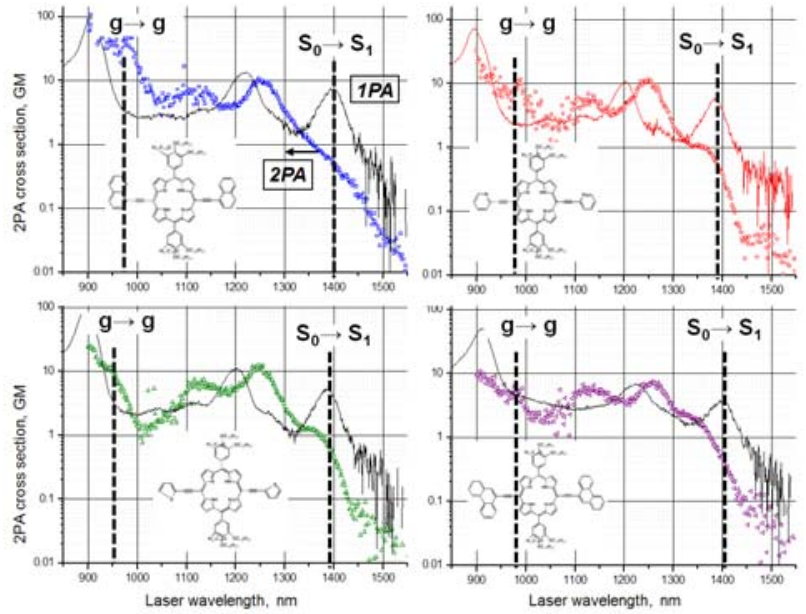
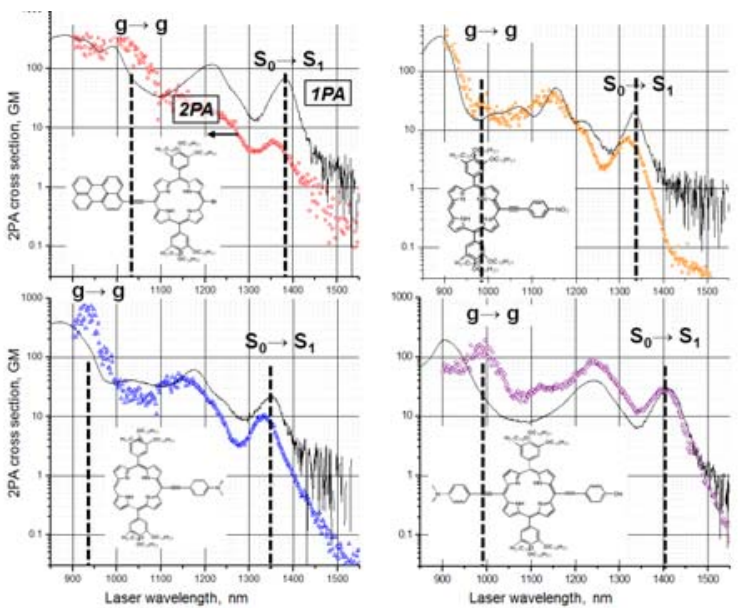

Fig. 12. 2PA spectra (symbols) and 1PA spectra (solid line) in eight enhanced solubility/enhanced 2PA meso-substituted porphyrins. Vertical lines indicate principal transitions from ground state to excited state.

3.3.3. Out next goal was to explore effect of fused porphyrin dimers on NLO propoperties [14]. We reported the synthesis of 7 new para-phenylene-bridged zinc porphyrin dimers (Anderson) with enhanced 2PA efficiency. A variety of links were tested for holding the paraphenylene bridges in $\pi$-conjugation with the porphyrin rings. Structure of these restraining links strongly influences the properties of the porphyrin dimers. The keto-linked dimer exhibits a long-lived singlet excited state and relatively strong fluorescence at $960 \mathrm{~nm}(1.7 \%$ quantum yield) in contrast to most previously reported conjugated porphyrin tapes, which are essentially non-emissive. Replacement of the cross-conjugated keto links by directly conjugated $\mathrm{C}-\mathrm{C}$ bonds eradicates the fluorescence and shifts the absorption maximum to $1077 \mathrm{~nm}$. On the other hand, replacement of the keto links with nonconjugated $\mathrm{CPh} 2$ links confers fluorescence at $736 \mathrm{~nm}$ (10\% quantum yield) and results in remarkably similar one- and 2-photon absorption behavior to that of mesomeso ethynylene-bridged porphyrin dimers (peak two-photon cross section: $\sigma_{2}=7,300 \mathrm{GM}$ at 878 $\mathrm{nm}$ ). Cross-conjugated keto links do more than just hold the para-phenylene bridge coplanar with the porphyrins; they reduce the HOMO-LUMO gap, although to a lesser extent than direct $\pi$ conjugated links. Planarized para-phenylene bridged porphyrin dimers provide insights into the relationship between previously investigated classes of conjugated porphyrin oligomers, and they open up possibilities for the synthesis of new types of near-IR two-photon absorbing dyes. Fig. 13 shows the linear extinction (a) and 2PA spectra (b) of fused dimer (dimer 6) and its non fused analog (dimer 2a). 

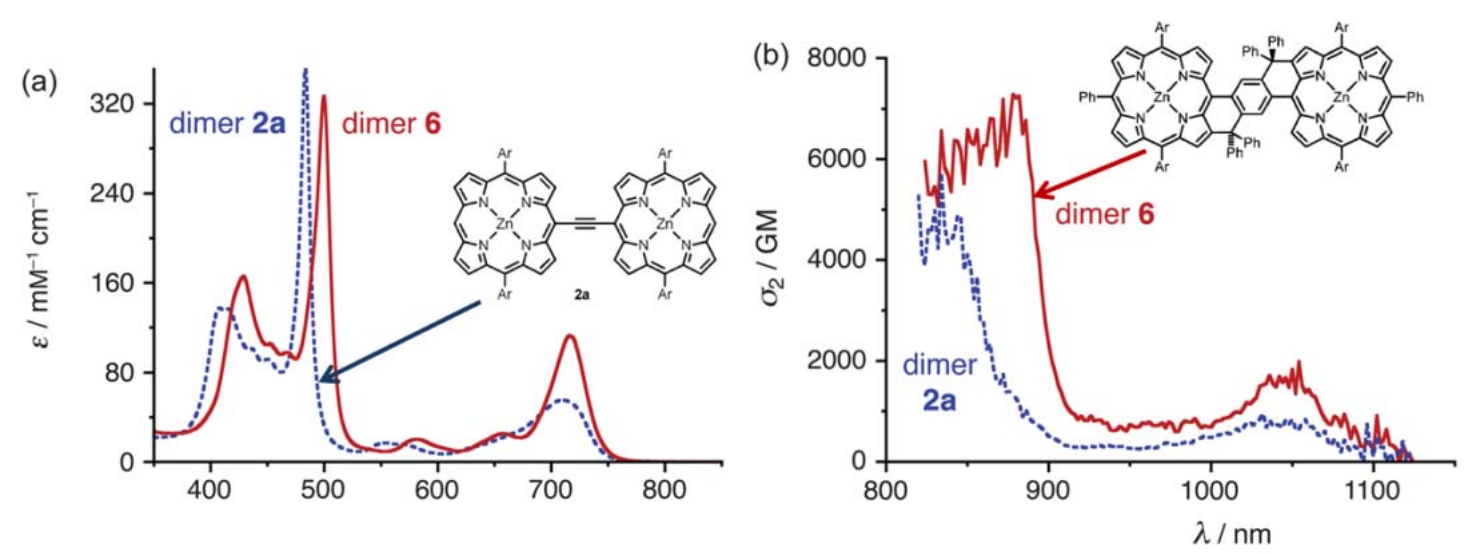

Fig. 13. Chemical structure (inserts), 1PA (panel a) and 2PA spectra (panel b) of fused dimer (dimer 6) and its non fused analog (dimer 2a).

3.3.3. Two-photon absorption in butadiyne-linked porphyrin dimers and torsional and substituent effects [15]. Eight $\pi$-extended bis-porphyrins possessing butadiyne-link were prepared using two synthetic strategies. Central unit was further extended via two additional arylethynyl moieties. All substituents were carefully designed to ensure both diverse electron effect and good solubility. We investigated structure-property relationships for optimising the performance of these meso-meso butadiyne-linked porphyrin dimers. The peak 2PA cross sectionsincreased from 3,000 GM to 17,000 GM by attaching terminal electron-withdrawing or -donating groups.
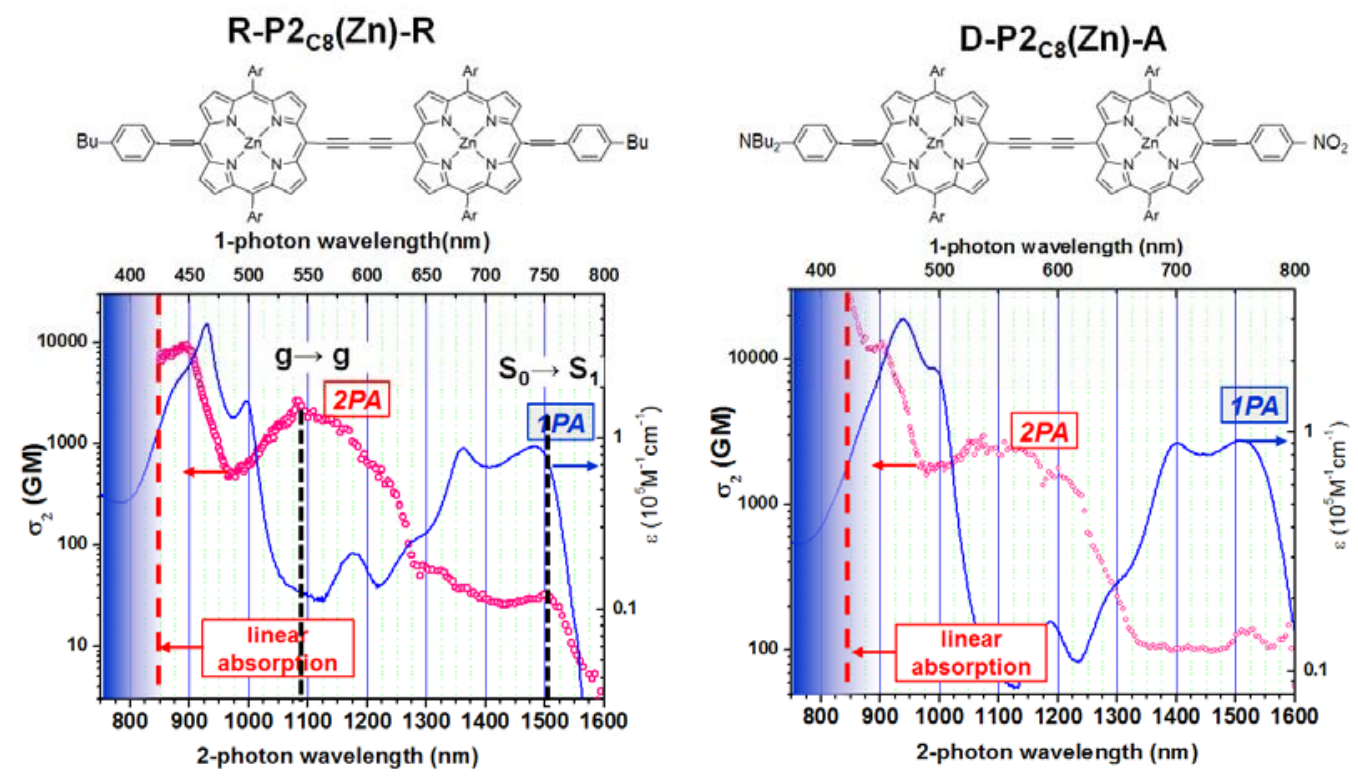

Fig. 14. Chemical structure (inserts), 1PA (solid line) and 2PA spectra (symbols) of conjugated dimers with neutral and asymmetrical push-pull donor-acceptor end groups.

We also investigated the influence of torsional angle about the central butadiyne-link on the 2PA spectra. The two-photon excited fluorescence spectra of porphyrin dimers in viscous media, under conditions such that excited states do not planarise prior to emission, reveal that dimers in planar conformations dominate the two-photon absorption. 


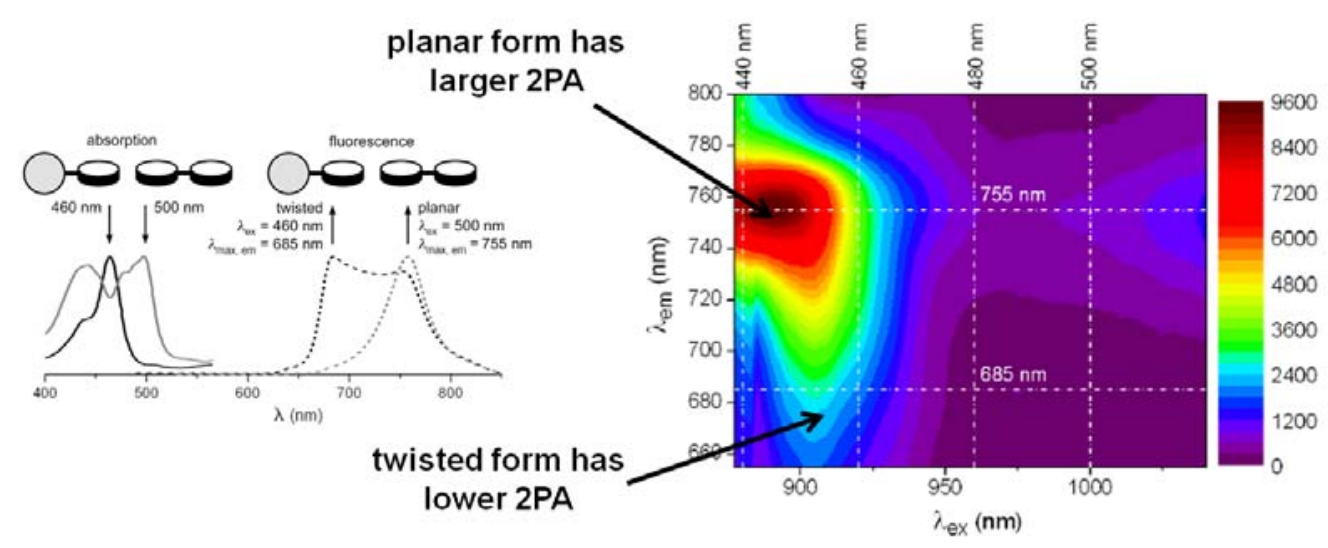

Fig. 15. Effect of torsion on the 1PA spectra (left panel) and 2PA spectra (right panel) of conjugated dimer R-P2 $\mathbf{C B}_{\mathbf{B}}(\mathrm{Zn})-\mathbf{R}$ in viscous chloroform/polymer solution. Right panel shows 3D two-photon excitation-emission plot.

3.4. New model for vibronic amplification of 2PA in non-symmetrical (dipolar) chromophores [16]. Previously it has been assumed that in non-symmetrical molecules, where the parity selection rules are relaxed, the 2PA and 1PA spectra should be closely related to each other. Even though this assumption is generally valid for the lowest energy 0-0 transition of dipolar organic chromophores, it has been observed that the vibronic distribution may still be quite different, which constitutes a long held puzzle for two-photon spectroscopy. Fig. 16 shows one such example where there is a marked difference in the $S_{0} \rightarrow S_{1}$ transition region (460-540 nm) of a common dye Rhodamine $\mathrm{B}$. In the 1PA spectrum the $0-0$ transition is stronger than the accompanying 0-1 vibronic peak, whereas in the 2PA spectra the situation is reversed, i.e. the 0-1 component is larger than the 0-0 component. Furthermore, the corresponding vibrational frequency is also slightly but distinctly different.
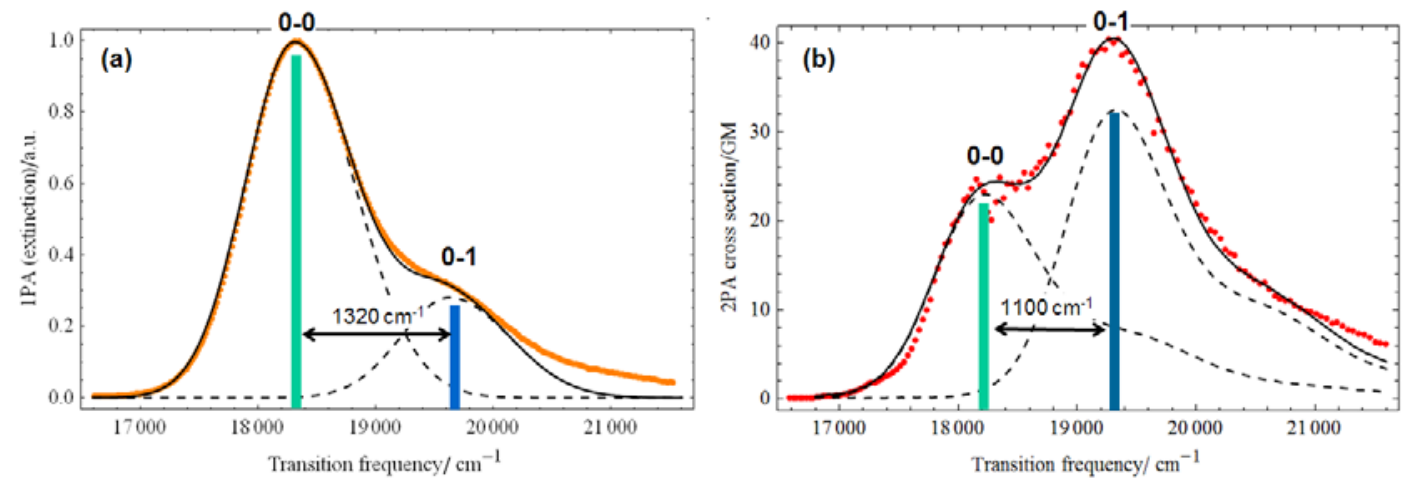

Fig. 16. One-photon absorption (a) and two-photon absorption (b) spectra in Rh. B in ethanol in the $\mathrm{S}_{0} \rightarrow \mathrm{S}_{1}$ transition region (460-540 nm). Experimental values are shown by orange line (a) and red dots (b); Vertical bars represent the relative contribution and the center frequencies of 0-0 and 0-1 components; Dashed lines in panel (a) are Gaussian fits to the 0-0 and 0-1 transitions to help determine Franc-Condon vibrational frequency. Dashed lines in panel (b) are replicas of the 1PA spectrum 
The relative intensity of the 0-0, 0-1, etc components of 1PA spectra (vibronic progression) are usually well explained by the so-called Frank-Condon (FC) factors, which are proportional to the overlap matrix elements between vibrational wave functions in the ground- and excited electronic state, while the transition dipole moment $\mu^{\mathrm{e}}$ (superscript e denotes electronic part of dipole) matrix element, is considered independent of the vibrational degrees of freedom (Condon approximation).

In the literature there is a well know example of benzene, where the vibronic component of the 2PA spectrum is amplified with respect to the $0-0$ component. This relative amplification occurs in benzene because due to symmetry selection rules the $2 \mathrm{PA}$ cross section vanishes for the $0-0$ component, while the 0-1 transition becomes partially 2PA-allowed. The last is explained because the transition dipole matrix elements connecting the ground state to vibrationally-excited levels in the excited electronic states may become slightly allowed by coupling to the vibrational coordinate (Herzberg-Teller or so-called non-Condon effects).

The origin of different vibronic intensities in non-centrosymmetrical chromophores has remained a puzzle because here the 2PA transition is already fully allowed, and it is thus reasonable to assume that a small additional contribution that may result from the Herzberg-Teller type interaction should not lead to any significant change of the 2PA vibronic progression. Indeed, if one assumes that the probabilities of 1PA and 2PA mostly depend on exactly the same transition matrix elements, then one would expect a very similar vibronic progression in both cases. Fig. 16 and several other experimental data suggest, however, that this is not the case.

Could there be some additional electronic molecular parameter that is coupled to vibrational degrees of freedom but which does not directly manifest in 1PA? We propose that this is the permanent electric dipole moment difference between the ground- and excited electronic states, $\Delta \mu^{\mathrm{e}}$. Our model assumes that the main contribution to the $2 \mathrm{PA}$ tensor $\left(S^{\mathrm{e}}\right)$ in the lowest dipole allowed transition is due to the factor:

$$
S^{e} \approx \Delta \mu^{e} \mu^{e},
$$

and that all other contributions (sum over all other states) are much smaller in value and may be discarded. According to Franck-Condon principle, $\mu^{\mathrm{e}}$ for dipole-allowed transition does not depend in the first approximation on vibrational coordinates. We argue that the $S^{\mathrm{e}}$ must then change through the dependence of $\Delta \mu^{\mathrm{e}}$ on vibrational coordinate $Q_{\mathrm{a}}$. Expanding $\Delta \mu^{\mathrm{e}}$ into the Taylor series up to the second (linear) term, gives:

$$
S^{e}\left(Q_{a}\right)=S^{e}\left(Q_{a}^{0}\right)+\left(\frac{\partial S^{e}}{\partial Q_{a}}\right)_{Q_{a}^{0}} Q_{a}=\mu^{e}\left(\Delta \mu^{e}\left(Q_{a}^{0}\right)+\left(\frac{\partial \Delta \mu^{e}}{\partial Q_{a}}\right)_{Q_{a}^{0}} Q_{a}\right),
$$

In other words, we apply the Herzberg-Teller type expansion not to the transition dipole moment, $\mu^{\mathrm{e}}$, (as was the case in benzene) but rather to the permanent dipole moment matrix element.

After taking the matrix element of $S^{\mathrm{e}}$ between vibrational wave functions of the ground and excited states, and taking the modulus squared, we arrive at the final expression for the 2PA spectrum for the transition between electronic states $g$ and $f$ (without taking into account possible interference between Frank-Condon and Herzberg-Teller terms): 
$\sigma_{2}(\omega)=A\left[\left|\Delta \mu^{e}\right|^{2}\left|\mu^{e}\right|^{2} \sum_{n} \delta\left(\omega_{f g}+\Omega_{k} n_{k}-\omega\right) F C_{n}+\left|\frac{\partial\left(\Delta \mu^{e}\right)}{\partial Q_{a}}\right|_{Q_{e q}}^{2} \frac{\hbar}{2 M_{a} \Omega_{a}}\left|\mu^{e}\right|^{2} \sum_{m_{k}} \delta\left(\omega_{f g}+\Omega_{k} m_{k}+\Omega_{a}-\omega\right) F C_{m_{k}}\right]$,

where $\sigma_{2}$ is the 2PA cross section, $\omega_{f g}$ is the optical transition frequency of the 1PA (0-0) maximum, $\omega$ is the twice laser frequency, $A$ is a constant, $\delta(x)$ is the delta function, $n_{k}$ is the number of excited vibrational quanta of mode $k, \Omega_{k}$ is the vibrational frequency of that mode, $F C$ is the Frank-Condon factor.

This formula can be now applied to quantitatively explain the amplification of the vibronic 2PA components in Rhodamine B and other non-symmetrical chromophores. According to eq. (3) the shape of the 2PA spectrum is described by the sum of replicas of the 1PA spectrum that are shifted by integer multiples $(0,1)$ of the characteristic HT vibrational frequency, $\Omega_{a}$. Fig. 16 (b) shows the theoretical 2PA spectrum that is obtained by superimposing two replicas of the 1PA spectrum (dashed curves). The result (solid black curve) shows a very good correspondence with the experimental 2PA spectrum (red dots). Comparable quantitative correspondence was obtained with different types of non-symmetrical chromophores including oxazines and fluorescent proteins.

\section{Personnel involved in the project:}

Prof. Aleksander Rebane (P.I)

Ass. Res. Prof. Mikhail Drobizhev

Dr. Galyna Dubinina (postdoctoral researcher shared 50/50 with Prof. Kirk Schanze UoF)

Dr. Yuryi Stepanenko (Visiting Research Professor, Polish Acad. Sci.)

Dr. Pawel Wnuk (Visiting Researcher, Polish Acad. Sci.)

Nikolay Makarov (graduate student)

Bret Davis (graduate student)

Alexandr Mikhailov (graduate student)

Geoffrey Wicks (graduate student)

Thomas Weas (undergraduate student, part time)

Jake Lindstrom (undergraduate student, part time)

Eric Beuerman (undergraduate student)

Kamlynn Thomas (undergraduate student)

\section{Publications in refereed journals:}

1. "Phenylene vinylene platinum(II) acetylides with prodigious two-photon absorption," G. G.

Dubinina, R. Price, K. A. Abboud, G. Wicks, P. Wnuk, Yu. Stepanenko, M. Drobizhev, A.

Rebane, K. S. Schanze, J. Am. Chem. Soc. 134(47), pp. 19346-19349 (2012).

2. "Modified p-phenylene vinylene platinum (II) acetylides with enhanced two-photon absorption in solid host," , Dubinina, Galyna G., Price, Randi S., Wicks, Geoffrey, Wnuk, Pawel, Stepanenko, Yuriy, Drobizhev, Mikhail, Rebane, Aleksander, Schanze, Kirk S., Proceedings of SPIE 8622, ORGANIC PHOTONIC MATERIALS AND DEVICES XV Edited by:Tabor, CE; Kajzar, F; Kaino, T; Koike, Y, 86220I ( 2013)

3. "Nonlinear absorption properties of platinum acetylide complexes and their optical power limiting materials," Price, Randi, Dubinina, Galyna, Shelton, Abigail, Rebane, Aleks, Schanze, Kirk, Abstracts ACS v. 245 pp. 440-INOR (2013). 
4. "Polymer monoliths containing two-photon absorbing phenylene vinylene platinum(II) acetylide chromophores for optical power limiting.” R.S. Price, G. Dubinina, G. Wicks, M. Drobizhev, A. Rebane, K. S. Schanze (submitted).

5. "Relation between two-photon absorption and dipolar properties in a series of fluorenyl-based chromophores with electron donating or electron withdrawing substituents," A. Rebane, M.

Drobizhev, N. S. Makarov, E. Beuerman, J. E. Haley, ,D. M. Krein, A. R. Burke, J. L. Flikkema, Th. M. Cooper, J. Phys. Chem A 115 (17), pp. 4255-4262 (2011).

6. "Symmetry breaking in platinum acetylide chromophores studied by femtosecond twophoton absorption spectroscopy," A. Rebane, M. Drobizhev, N. S. Makarov, G. Wicks, P. Wnuk, Yu. Stepanenko, J. E. Haley, D. M. Krein, J. L. Fore, A. R. Burke, J. E. Slagle, D. G. McLean, T. M. Cooper, JPC A 118 ( 21) pp 3749-3759 (2014).

7. "Direct synthesis of 2,5-bis(dodecanoxy) phenyleneethynylenebutadiynes by Sonogashira coupling reaction,” E. Arias, I. Moggio, R. Torres, R. Ziolo, J.-L. Maldonado, K. Green, T. Cooper, G. Wicks, A. Rebane, M. Drobijev, N. Makarov, M. Ottonelli, G. Dellepiane European J. of Org. Chem. 24, p. 5341-5352 (2013).

8. "Photophysics and non-linear absorption of $\mathrm{Au}(\mathrm{I})$ and $\mathrm{Pt}(\mathrm{II})$ acetylide complexes of a thienylcarbazole chromophore," S. Goswami, G. Wicks, A. Rebane and K. S. Schanze, Dalton Transactions (in print).

9. "2-photon absorption propoerties of platinum capped phenylene ethynylene oligomers," Julia M. Keller, G. Wicks, A. Rebane, Kirk S. Schanze (in preparation).

10. "Stereochemical effects on two-photon absorbing platinum acetylides," A. H. Shelton, R.S. Price, C. F. Campana, K. Abboud, G. Wicks,M. Drobizhev, A. Rebane,K. S. Schanze (in preparation).

11. "Amplified two-photon absorption in trans-A2B2-porphyrins bearing nitrophenylethynyl substituents," Agnieszka Nowak-Król, Craig J. Wilson, Mikhail Drobizhev, Dmitry V.

Kondratuk, A. Rebane, Harry L. Anderson, Daniel T. Gryko, ChemPhysChem, 13(17), 39663972 (2012).

12. "Synthesis and linear and nonlinear optical properties of low-melting $\pi$-extended porphyrins," Dominik Koszelewski, Agnieszka Nowak-Krol, Mikhail Drobizhev, Craig J. Wilson, Joy E.

Haley, Thomas M. Cooper, Jerzy Romiszewski, Ewa Gorecka, Harry L. Anderson, Aleksander Rebane and Daniel T. Gryko, J. Mater. Chem. C, 1, pp. 2044-2053 (2013).

13. "Strong two-photon absorption enhancement in a unique bis-porphyrin bearing a diketopyrrolopyrrole unit." Nowak-Krol, Agnieszka, Grzybowski, Marek, Romiszewski, Jerzy, Drobizhev, Mikhail, Wicks, Geoffrey, Chotkowski, Maciej, Rebane, Aleksander, Gorecka, Ewa, Gryko, Daniel T. Chemical Communications, 49(75) pp. 8368-8370 (2013).

14. "Engineering conjugation in para-phenylene-bridged porphyrin tapes," M. Pawlicki, M.

Morisue, N. K. S. Davis, D. G. McLean, J. E. Haley, E. Beuerman, M. Drobizhev, A. Rebane, A. L. Thompson, S. I. Pascu, G. Accorsi, N. Armaroli, H. L. Anderson, Chemical Science, 3(5), pp. 1541-1547 (2012).

15. "Two-photon absorption in butadiyne-linked porphyrin dimers: Torsional and substituent effects," J. D. Wilkinson, G. Wick, A. Nowak-Król,, Ł. G. Łukasiewicz, C. J. Wilson, M. Drobizhev, A. Rebane, D.T. Gryko, H. L. Anderson, J. Mat. Chem C (submitted).

16. "Describing two-photon absorptivity of fluorescent proteins with a new vibronic coupling mechanism," M. Drobizhev, N. Makarov, S. Tillo, Th. E. Hughes, A. Rebane, J. Phys. Chem. B, 116 (5), pp. 1736-1744 (2012). 
17. "Optimizing simultaneous two-photon absorption and transient triplet-triplet absorption in platinum acetylide chromophores", K. Schanze, Kim, Kye-Young; A. Shelton, M. Drobizhev, N. Makarov, A. Rebane, J. Phys. Chem. A, 114 (26), 7003-7013 (2010).

18. "Alternative selection rules for one- and two-photon transitions in tribenzotetraazachlorin: Quasi-centrosymmetrical $\pi$-conjugation pathway of formally non-centrosymmetrical molecule," N.S. Makarov, M. Drobizhev, G. Wicks, E. A. Makarova, E. A. Lukyanets, and A. Rebane, J. Chem. Phys. 138, 214314 (2013).

19. "One-photon photophysics and two-photon absorption of 4-(9,9'-Di(2-ethylhexyl)-7diphenylaminofluoren-2-yl)-2,2':6',2'-terpyridine platinum complexes with large two-photon absorption," Zhiqiang Ji, Yunjing Li, T. M. Pritchett, N.S. Makarov, J. E. Haley, A. Rebane, Wenfang Sun, Chem A Euro J. 17 (8), pp. 2479-2491 (2011).

20. "Relation between two-photon absorption and dipolar properties in a series of fluorenyl-based chromophores with electron donating or electron withdrawing substituents," A. Rebane, M. Drobizhev, N.S. Makarov, E. Beuerman, J. E. Haley, D. M. Krein, A. R. Burke, J. L. Flikkema, Th. M. Cooper, JPC A 115 (17), pp. 4255-4262 (2011).

\section{Interactions/Transitions:}

\section{a. Participation/presentations at meetings, conferences, seminars, etc.}

1. N. S. Makarov, M. Drobizhev, E. A. Makarova, E. A. Lukyanets, A. Rebane, "Quantitative nonlinear spectroscopy of free-base tribenzo-tetraazachlorin", MSU 2010 Student Research Celebration, 8 April 2010.

2. (Talk) N. S. Makarov, M. Drobizhev , E. A. Makarova, E. A. Lukyanets, A. K. Rebane, "Two-photon spectroscopy allows for probing of the pi-conjugated pathway in tetrapyrroles", Optics and Photonics 2010, 1-5 August, 2010, San Diego, CA.

3. (Talk) M. Drobizhev, N. S. Makarov, S. E. Tillo, T. E. Hughes, A. K. Rebane, "HerzbergTeller vibronic contribution to mesomeric dipole moment determines two-photon absorptivity of fluorescent proteins", Photonics West 2011, 22-27 January, 2011, San Francisco, CA.

4. (Talk) A. Rebane, M. Drobizhev, N. S. Makarov, E. B. Beuerman, T. M. Cooper, J. E. Rogers-Haley, D. G. McLean, J. E. Slagle, D. M. Krein, J. L. Monahan, A. R. Burke, A. Fratini, "Effects of ligand on two-photon absorption of platinum acetylide complexes", Photonics West 2011, 22-27 January, 2011, San Francisco, CA.

5. M. Drobizhev, N. S. Makarov, S.Tillo, T. E. Hughes, A. Rebane, "Herzberg-Teller vibronic contribution to mesomeric dipole moment determines two-photon absorptivity of fluorescent proteins", Biophysical Society Meeting, March 5-9, 2011 Baltimore.

6. A. Rebane, N. Makarov, Erich Beuerman, M. Drobizhev, Describing two-photon absorptivity of (Zwitter)ionic fluorochromes and fluorescent proteins with a new vibronic coupling mechanism,", SPIE Photonics West, 25-27 January, 2012, San Francisco.

7. A. Rebane, M. Drobizhev, "Advanced methods of femtosecond two-photon absorption spectroscopy," AFOSR Workshop on, April 21-22, 2011 Dayton, OH

8. A. Rebane, M. Drobizhev," Quantitative femtosecond two-photon absorption spectroscopy: from nonlinear absorbers to all-optical molecular voltmeter," 20th International Laser Physics Workshop, July 11-15, 2011, Sarajevo, Bosnia and Herzegovina.(Invited talk)

9. A. Rebane, M. Drobizhev, "Two-Photon Absorption Spectroscopy: From Nonlinear Absorbers to All-Optical Molecular Voltmeter," MRS Fall Meeting, November 28 December 2, 2011 (Invited talk) 
10. (Invited) "Femtosecond two-photon fluorescence excitation and nonlinear transmission spectroscopy of platinum acetylide complexes and related chromophores," A. K. Rebane, M. Drobizhev, G. Wicks, A.Mikhailov, Th. M. Cooper, Yu. Stepanenko, P. Wnuk, SPIE Optics \& Photonics, Aug. 25 - 29, 2012, San Diego, CA.

11. (Invited) "Vibronic coupling in two-photon absorption of dipolar chromophores," ) 11th Int. Conf. Spectral Hole Burning Single Molecule Spectroscopy, August 27-30, 2012, Tübingen, Germany.

12. (Invited) A. Rebane, M. Drobizhev, Daniel T. Gryko, Harry Anderson, Jean Starkey, "Twophoton absorption spectroscopy and applications of tetrapyrroles." 7th International Conf. Porphyrins \& Phthalocyanines, July 1 -6, 2012, Jeju, South Korea.

13. (Talk) "All-optical method of sensing the components of the internal local electric field in proteins,” M. Drobizhev, J. N. Scott, P. R. Callis, and A. Rebane, SPIE Photonics West, February 2 - 7, 2013, San Francisco, CA.

14. (Talk) "Modified p-phenylene vinylene platinum (II) acetylides with enhanced two-photon absorption: solution vs solid host," G. G. Dubinina,R. Price, K. A.Abboud, G. Wicks, P. Wnuk, Yu.Stepanenko, M. Drobizhev, A. Rebane, K.S. Schanze, SPIE Photonics West, February 2 - 7, 2013, San Francisco, CA.

\section{b. Consultative and advisory functions to Air Force and other DoD laboratories.}

1. Mini-symposium presentations at WPAFB, May 12, 2010. Dr. Aleks Rebane: "Intrinsic (femtosecond) two-photon absorption: methods, materials and applications" and "Numerical simulation of propagation of ultrashort pulse in two-photon absorbing media";

2. Collaborated with Dan McLean on developing numerical calculation of dispersion of index of refraction in dye-doped polymer based on measured linear extinction spectra and using KramerKronig relations;

3. Performed measurements of the 2PA spectra and 2PA cross sections in an extended series of AFX compounds provided by Dr. Seng Tan (RXBN). This project is now funded separately as 1 yr subcontract from UES Project No. P875-4;

4. We have carried out preliminary measurements of 2PA in the samples containing AFX chromophores superimposed on gold nanoparticle structures sent to us by Dr. Augustine Urbas USAF AFMC AFRL/RXPJ;

1. WPAFB/AFRL Mini-symposium presentation, April 21-22, 2011. Dr. Aleks Rebane: "Advanced methods of femtosecond two-photon spectroscopy";

2. Provided Dan McLean with an improved template for numerical calculation of dispersion of index of refraction in dye-doped polymer based on the measured linear extinction spectra; 3. Continued performed measurements of the 2PA spectra and 2PA cross sections in AFX compounds provided by Dr. Seng Tan $(\mathrm{RXBN})$. This project is funded separately as a continuing subcontract from UES (Project No. P875-4);

4. We have carried out preliminary measurements of 2PA in the samples containing AFX chromophores superimposed on gold nanoparticle structures sent to us by Dr. Augustine Urbas USAF AFMC AFRL/RXPJ;

WPAFB/AFRL Mini-symposium presentation, July 26-27, 2012. Dr. Aleks Rebane: "Comprehensive approach to femtosecond 2PA spectroscopy: Fluorescence detection and nonlinear transmission”; 
2. Continued performed measurements of the 2PA spectra in AFX series compounds provided by Dr. Seng Tan $(\mathrm{RXBN})$. This project is funded separately as a continuing subcontract from UES (Project No. P875-4);

DoD contacts: Dr. Thomas Cooper (group leader), Dr. Joy E. Haley, Dr. Ruth Pachter, Dr. Daniel McLean, Dr. Augustine Urbas (former group leader), AFRL/MLPJ Bldg 651, 3005 Hobson Way, Wright-Patterson AFB, OH 45433-7702

Phone (937) 255-3808 x3179, Fax (937) 255-1128.

\section{c. Transitions to technology application.}

7. New discoveries, inventions, or patent disclosures. (If none, report None.)

None

8. Honors/Awards:

(a) Honors and awards received during the grant/contract period.

(b) Lifetime achievement honors prior to this effort:

2010 Russian Academy of Sciences Yu. N. Denisyuk Holography Medal

2004 Montana State University Cox Meritorious Research Award

1999 Charles and Nora Wiley Award of the Montana State University.

1996 Leopold Ruzicka Prize of the Swiss Federal Institute of Technology

1993 International Commission for Optics Prize 